\title{
INTROGRESSION FROM DIPLOID AEGILOPS SPELTOIDES TO TETRAPLOID DURUM WHEAT*
}

\author{
ALIZA VARDI \\ Laboratory of Genetics, The Hebrew University, Jerusalem, Israel
}

Received 30.iii.70

\section{INTRODUCTION}

IN a previous paper (Vardi and Zohary, 1967) diploid-to-tetraploid introgression was reported in wheats. It was demonstrated that triploid interspecific hybrids serve as effective " bridges" for gene-transfer, first when the tetraploid receptor and the diploid donor share a common genome $(\mathcal{T}$. durum $\times T$. boeoticum $\mathrm{AAB}$ genomic combination), and second when an alien diploid Aegilops species hybridises with the tetraploid wheat (T.durum $\times A e$. longissima combination).

The present paper supplements the previous findings. It deals with the process of introgression from Aegilops speltoides, the alleged diploid contributor of B genome to polyploid wheats (Sarkar and Stebbins, 1956; Riley et al., 1958). Introgression via the $T$. durum $\times$ Ae. speltoides ABB triploid is complicated cytogenetically by the fact that the introduction of $A e$. speltoides chromosomes suppresses the diploidisation effect of chromosome $5 \mathrm{~B}$ of the wheats (Riley and Chapman, 1964; Kimber, 1966; Riley et al., 1968). This affects the stabilisation of hybrid derivatives so that the process of introgression form $A e$. speltoides to $\mathcal{T}$. durum is somewhat unique and merits special consideration.

\section{Methods}

Meiosis in P.M.C.s served both for determination of chromosome numbers and for the study of chromosome pairing. Anthers were fixed in $3: 1$ alcohol-acetic acid for 24 hours, stored in 70 per cent. alcohol and stained in acetocarmine.

Since chromosome numbers varied from plant to plant pairing was not expressed in values of " chiasmata per cell"; instead the values of " associations per chromosome" were used, i.e. twice the number of chiasmata per cell divided by the number of chromosomes in the examined plant. In the parental lines, the $F_{1}$ triploid and the majority of $F_{3}$ plants, this value is based on examination of 30 randomly picked mataphases. In $F_{2}$ plants and in several $\mathrm{F}_{3}$ individuals fewer cells (three or more) have been analysed. We found that with the large number of chromosomes present, variation in chiasmata numbers between cells within plants was relatively small and the sampling error due to samples of only few cells negligible for the purpose of the present work.

Pollen fertility was determined by dissecting mature anthers soaked in 4 per cent. acetocarmine and scoring c. 500 pollen grains per plant. Grains were considered normal when they were rounded and well stained.

* The material presented in this paper comprises a part of the Ph.D. thesis presented to the Hebrew University, Jerusalem. 
Seed fertility was determined by examination of the two lower florets in the spikelet. A floret was considered fertile if a well-developed kernel was found in it. In the case of more fertile plants a sample of 100 florets (i.e. 50 spikelets) was employed. In the semi-sterile plants, and particularly in the triploids themselves, seed set was determined by examination of all available spikes.

\section{Experimental procedures AND RESUlts}

Artificially produced $T$. durum $\times$ Ae. speltoides triploid hybrids were planted intermixed with their parental durum wheat line ( $\mathcal{T}$. durum cultivar Nursit 163). Consequently, the male sterile $F_{1}$ hybrids were massively exposed to back-cross pollination by their tetraploid wheat parent. The occasional back-cross seeds produced by the largely sterile triploids were planted in 1966 and the second generation of hybrid derivatives was grown and analysed. The $F_{2}$ plants too were interplanted with tetraploid wheat. But since some $\mathrm{F}_{2}$ plants were already semi-fertile, the seed they produced was apparently a mixture of selfed and second back-cross products. Selected families of the third hybrid generation derivatives were grown in 1967. The following diagram sketches the experimental design employed:

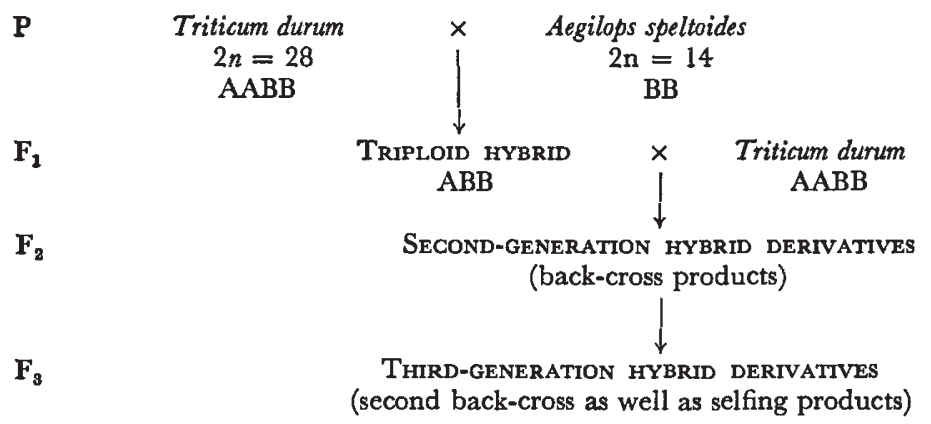

The results obtained can be summarised as follows:

(a) $F_{1}$ hybrids

Three T. durum $\times$ Ae. speltoides triploid $(2 n=21)$ plants (genomic constitution $\mathrm{ABB}$ ) were used. All showed considerable vegetative vigour under Jerusalem nursery conditions and exceeded both parents in number of tillers. Meiosis (table 1) was similar in all three hybrids. There were four to seven bivalents in metaphase I and frequent trivalents. In some P.M.C.s as many as five trivalents were observed. This relatively high number of trivalents is ascribed by us to suppression of the 5B diploidisation effect by Ae. speltoides chromosomes (Riley and Chapman, 1964). Thus chromosome pairing in the $A B B$ triploids differed considerably from that of the $T$. durum $\times T$. boeoticum AAB combination previously explored (Vardi and Zohary, 1967). Homoeologous pairing in the durum $\times$ speltoides hybrids is also indicated (table 1) by relatively high " association per chromosome " values.

Triploid ABB plants were completely male sterile, with non-dehiscing anthers and pollen abortion of 98-99 per cent. With the massive exposure to T. durum pollen, back-cross was made possible. A total of about 1800 well- 
developed spikelets were harvested from the three $F_{1}$ plants. They contained 11 back-cross seeds from which nine second-generation hybrid derivatives were grown to maturity (table 1).

\section{(b) Second-generation hybrid derivatives}

There was considerable morphological variation, particularly in ear shape (plate I) in the nine second-generation plants. In their semi-spreading growth habit almost all resembled the $F_{1}$ rather than the $T$. durum parent. Eight $F_{2}$ plants contained 27 to 29 chromosomes (table 1). The chromosome number in the ninth plant was 25 . In other words, there is an increase in chromosome numbers towards the tetraploid level in the $F_{2}$ plants secured from the ABB triploids. Furthermore, associated with this crude attainment of tetraploidy is a considerable improvement in chromosome pairing. The $F_{2}$ plants containing 27 to 29 chromosomes formed 10 to 12 bivalents, as well as frequent trivalents or quadrivalents. None, however, achieved complete cytological stabilisation. Even the $2 n=28 \quad \mathrm{~F}_{2}$ plant showed some pairing irregularities (table 1).

Parallel to the behaviour during meiosis, the second-generation plants showed considerable pollen abortion and seed sterility. Only two $\mathrm{F}_{2}$ individuals (one with $2 n=27$, and the second with $2 n=28$ ) had more than 15 per cent. normally stained pollen. Only in these two plants did anthers partially dehisce, and seed set in them was about 10 per cent. Consequently, third-generation derivatives could be raised from them. The other seven $F_{2}$ plants were completely sterile.

\section{(c) Third-generation derivatives}

Two $F_{3}$ families of nine sibs each were grown. In vegetative vigour $F_{3}$ plants exceeded the $F_{2}$ counterparts. In their general morphology, and particularly in their ear shape, plants were more similar to $T$. durum than their $F_{2}$ parents were. Considerable morphological variation was noticed between and within families. Chromosome numbers in $\mathrm{F}_{3}$ varied between $2 n=27$ and $2 n=29$ with an apparent tendency towards stabilisation at the euploid number of $2 n=28$ (table 2). Also chromosome pairing was markedly improved as compared with the $F_{2}$ generation. But pairing values characteristic of tetraploid wheat, i.e. 1.8-2.0 " associations per chromosome" "were approached by a single plant (No. 458B2-10) only. Moreover, $\mathrm{F}_{3}$ plants formed also occasional univalents, and one or even two trivalents or quadrivalents were observed in most plants (table 2).

The majority of progeny derived from the $2 n=29$ parent (458B2 family) had more than 16 per cent. stainable pollen and anthers partially dehisced. Pollen fertility in the second family was somewhat lower. Both families show partial fertility restoration as compared with the $F_{2}$ generation and numerous seed could be secured from both. This family also showed some increase in seed set. In the family derived from the $2 n=27 \mathrm{~F}_{2}$ parent (457A5 family) fertility was lower.

\section{Discussion}

Diploid to tetraploid introgression in wheat differs considerably in the cases of the two progenitors. In $T$. durum $\times T$. boeoticum AAB combination (Vardi and Zohary, 1967) only the two A sets pair regularly, while the 


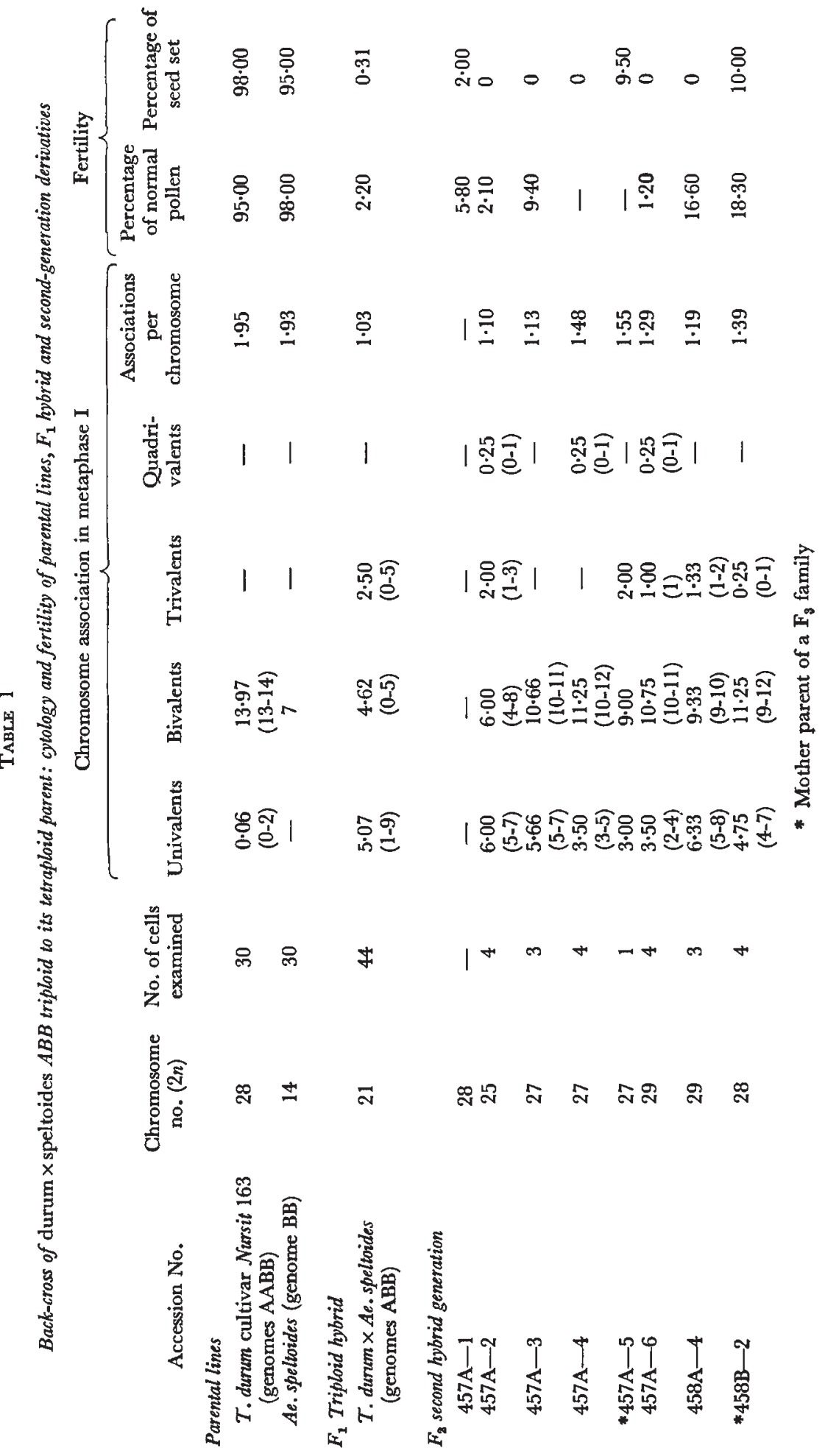




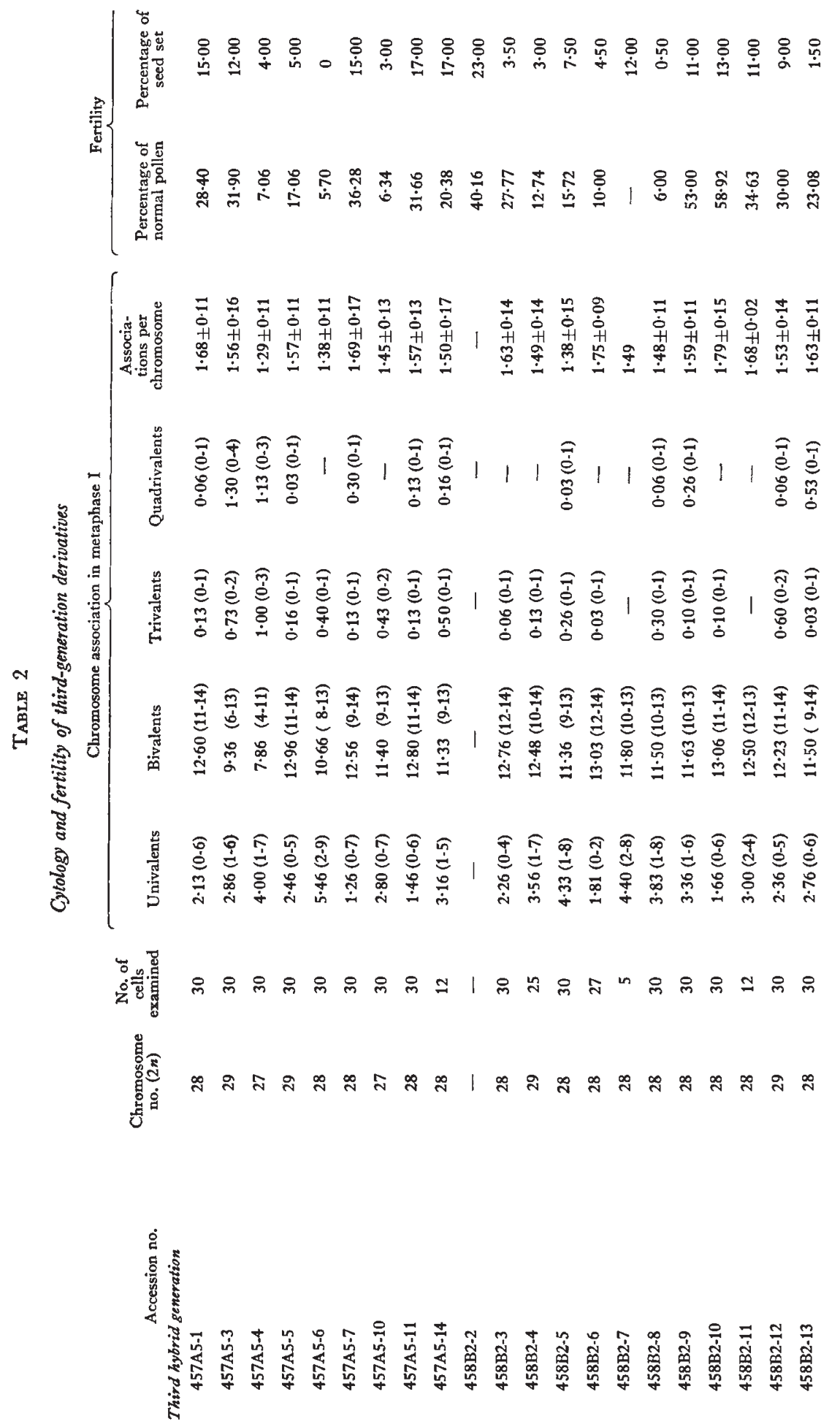


chromosomes of the single set of $\mathrm{B}$ genome usually appear as univalents. In contrast, in $T$. durum $\times$ Ae. speltoides $\mathrm{ABB}$ combination pairing is complicated by the suppression of the wheats' B5 effect (Riley and Chapman, 1964; Riley et al., 1968) and formation of trivalents is frequent. Exchanges between homeologous chromosomes frequently take place at the time of gamete formation in the $\mathrm{ABB}$ plants. They apparently account for the relatively lower production of stable or harmonious products by the $A B B$ triploids. In other words, chances for $7 \mathrm{~B}$ by $7 \mathrm{~B}$ segregation in anaphase $\mathrm{I}$ in the $\mathrm{ABB}$ triploid are less than the chances for $7 \mathrm{~A}$ by $7 \mathrm{~A}$ segregation in the $\mathrm{AAB}$ combination. This is probably the main reason for the relatively low fertility scored in the $\mathrm{ABB}$ triploid compared with their $\mathrm{AAB}$ counterparts.

In both $A A B$ and $A B B$ combinations the second generation back-cross products show an increase or "jump" in chromosome numbers-towards tetraploidy. The viable progeny secured from the triploids (with chromosomes numbers 27-29) already contain roughly $\mathrm{AABB}$ chromosome constitution. But in $\mathrm{ABB}$ combination considerable number of trivalents are still present in many plants. This indicates that full structural homozygosity or the regular control of bivalent formation were not yet achieved. Thus fertility in $\mathrm{F}_{2}$ was low and most of the $\mathrm{F}_{2}$ plants were sterile.

An obvious trend towards cytological stabilisation and restoration of fertility is already apparent in the third generation. None of the $F_{3}$ products is yet fully fertile, but the majority are already euploid. Chromosome pairing too is much improved. Another point worth mentioning is the discrepancy between pollen fertility and seed set data. As seen from table 2, the percentage of stainable pollen in most $F_{3}$ plants is considerably higher than the percentage of seed set. This might mean that selection of unbalanced recombinations is more severe in the early sporophytic stages, as compared with the gametophytic stage.

In summation, one is led to the conclusion that gene transfer from diploid Ae. speltoides to tetraploid $T$. durum is more complicated than the parallel introgression from diploid T. boeoticum. It is clear, however, that some of the $\mathrm{F}_{3}$ plants obtained are already roughly stabilised durum-like tetraploids. Another back-cross would probably lead to fully stabilised introgression products. In spite of the complications due to $5 \mathrm{~B}$ effect, speltoides to durum introgression via a triploid bridge is feasible, and the process can be utilised also in practical wheat breeding.

\section{Summary}

1. Triploid hybrids between Triticum durum (genomic constitution AABB) and Aegilops speltoides (BB) were found to set rare seed when massively backpollinated to their tetraploid durum parent. Roughly stabilised durum-like tetraploid derivatives were obtained in the third hybrid generation.

2. In the case of Ae. speltoides diploid to tetraploid introgression is complicated by the suppression of the wheats' $5 \mathrm{~B}$ effect on bivalent formation. Thus cytological stabilisation and restoration of fertility in $\mathrm{F}_{2}$ and $\mathrm{F}_{3}$ are relatively slower, compared with $T$. durum $\times T$. boeoticum $\mathrm{AAB}$ triploid combination.

Acknowledgments. - The author is indebted to Dr D. Zohary for his help and advice. Thanks are due to the Agricultural Research Service of the United States Department of Agriculture for a research grant (FG-Is-129) which partly supported this study. 


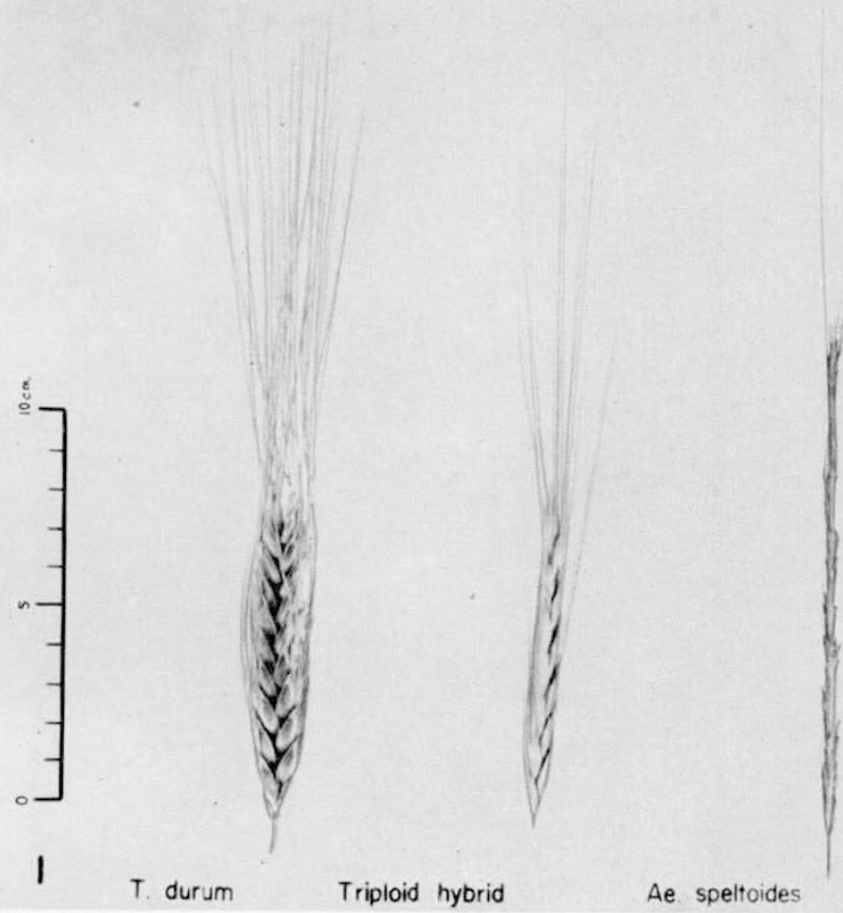

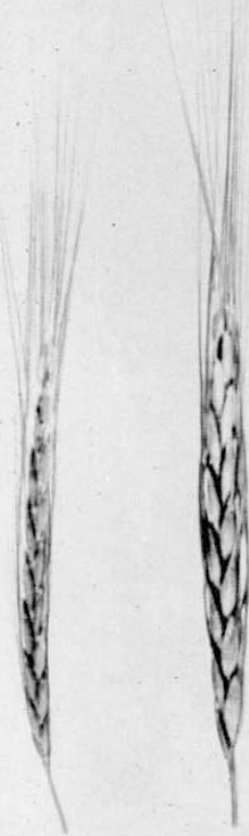

2

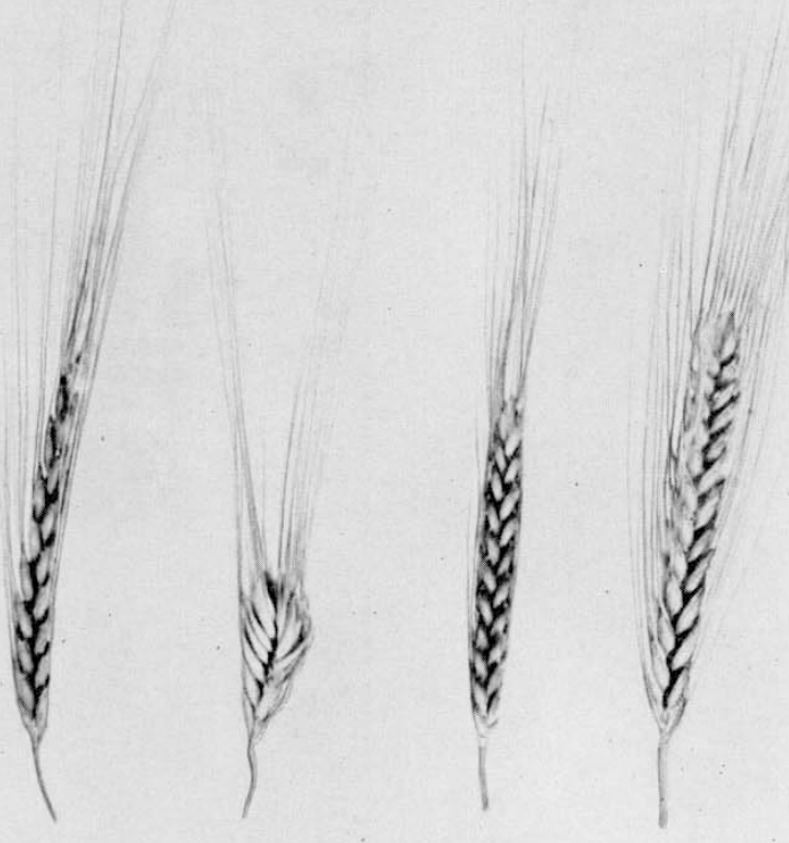

457 - A

First bockcross generation

to tetroploid wheot

Representative spikes from the parental species, $F_{1}$ triploid hybrid and the backcross derivatives in $T$. durum $\times$ Ae. speltoides $\mathrm{ABB}$ combination. 


\section{REFERENGES}

KIMBER, G. 1966. Estimate of the number of genes involved in the genetic suppression of the cytological diploidization of wheat. Nature, 212, 317.

RILEY, R., UNRAU, J., AND CHAPMAN, v. 1958. Evidence on the origin of the B genome of wheat. 7. Hered., 49, 91-99.

RILEY, R., AND CHAPMAN, v. 1964. Cytological determination of the homoeology of chromosomes of Triticum aestivum. Nature, 203, 156-158.

RILEY, R., CHAPMAN, V., AND JOHNSON, R. 1968. Introduction of yellow rust resistance of Aegilops comosa into wheat by genetically induced homoeologous recombination. Nature, 217, 383-384.

SARKAR, P., AND STEBBINS, G. L. 1956. Morphological evidence concerning the origin of the B genome of wheat. Am. 7. Bot., 43, 297-304.

VARDI, A., AND ZOHARY, D. 1967. Introgression in wheat via triploid hybrids. Heredity, 22, 541-560. 